V. Barucci

Nagoya Math. J.

Vol. 113 (1989), 99-119

\title{
A LIPMAN'S TYPE CONSTRUCTION, GLUEINGS AND COMPLETE INTEGRAL CLOSURE
}

\author{
VALENTINA BARUCCI
}

\section{§. Introduction}

Given a semilocal 1-dimensional Cohen-Macauly ring $A$, J. Lipman in [10] gives an algorithm to obtain the integral closure $\bar{A}$ of $A$, in terms of prime ideals of $A$. More precisely, he shows that there exists a sequence of rings $A=A_{0} \subset A_{1} \subset \cdots \subset A_{i} \subset \cdots$, where, for each $i, i \geq 0$, $A_{i+1}$ is the ring obtained from $A_{i}$ by "blowing-up" the Jacobson radical $\mathscr{R}_{i}$ of $A_{i}$, i.e. $A_{i+1}=\cup_{n}\left(\mathscr{R}_{i}^{n}: \mathscr{R}_{i}^{n}\right)$. It turns out that $\cup\left\{A_{i} ; i \geq 0\right\}=\bar{A}$ (cf. [10, proof of Theorem 4.6]) and, if $\bar{A}$ is a finitely generated $A$-module, the sequence $\left\{A_{i} ; i \geq 0\right\}$ is stationary for some $m$ and $A_{m}=\bar{A}$, so that

$$
A=A_{0} \varsubsetneqq A_{1} \varsubsetneqq \cdots \varsubsetneqq A_{m}=\bar{A} .
$$

In [15] G. Tamone studies when in the Lipman's sequence $(+) A_{i}$ is a "glueing of primary ideals of $A_{i+1}$ over a prime ideal of $A$ " (see [14] for definition). She shows in particular that $A_{i}$ is not always a glueing of primary ideals of $A_{i+1}$.

In this paper we give an algorithmic construction, for a Noetherian domain $A$ of any dimension, such that $\bar{A}$ is a finitely generated $A$-module, defining a new sequence $\left\{A_{i} ; i \geq 0\right\}$ of overrings of $A ; A_{i+1}$ is obtained from $A_{i}$, taking the dual of a distinguished radical ideal of $A_{i}$. We show that such a sequence is stationary for some $m, A_{m}=\bar{A}$ (cf. Theorem 1.8), and $A_{i}$ is always a glueing of primary ideals of $A_{i+1}$ (cf. Proposition 2.7 and Remark 2.2, a)).

A similar sequence was considered in [17] by $\mathrm{K}$. Yoshida in the case of a Noetherian ring satisfying the $S_{1}$-condition. As a matter of fact, the intermediate rings of the Yoshida sequence are defined in a rather different way, but the prime ideals occuring in their definition are linked to those that we use in our sequence (cf. for more details Remark 1.7).

Received June 2, 1987. 
However our result holds in a more general situation which turns out to be its natural context, that is $A$ is just a Mori domain. We recall that a Mori domain is a domain such that the ascending chain condition holds for integral divisorial ideals (e.g. Noetherian and Krull domains are Mori; for other examples and further properties of these domains cf. [11, $12,13,2,4])$. In this case the sequence of overrings of $A$ is stationary at $A^{*}$, the complete integral closure of $A$ (for a Noetherian domain, it coincides with $\bar{A}$, the integral closure of $A$ ).

In Section 2 we study the general procedure in order to descend along the sequence $\left\{A_{i} ; \mathrm{i} \geq 0\right\}$ constructed above. This procedure consists in a "contraction of ideals of $A_{i+1}$ over prime ideals of $A_{i}$ " (cf. Definition 2.1), that, in the Noetherian case, coincides with the glueing of primary ideals, as defined by G. Tamone in [14].

With the additional hypothesis that in our sequence $\left\{A_{i} ; i \geq 0\right\}$ the conductor of $A_{i}$ in $A_{i+1}$ is a radical ideal of $A_{i+1}$, for each $i$ (cf. Section 3 ), we show that the "contraction" coincides exactly with the glueing (of prime ideals), as defined by F. Ischebeck in [9]. Under this particular hypothesis, in the Noetherian case, we get a new characterization of seminormal domains (cf. Theorem 3.8); an analogous characterization, involving conductor jdeals, was given by $\mathrm{K}$. Yoshida, using his sequence (cf. [17, Theorem 2.2]). On the other hand, if the domain $A$ is not Noetherian, but Mori, we obtain a natural extension of the notion of seminormal domain (not in the integral closure but) in its complete integral closure: similarly to Traverso's result for Noetherian seminormal rings, (cf. [16, Theorem 2.1]) such a domain $A$ is obtained from its complete integral closure $A^{*}$ (that is a Krull domain) with a finite number of glueings over prime ideals of $A$ of a certain type (cf. Corollary 3.7). The paper ends with some examples of Mori, non-Noetherian domains of this kind.

Throughout the paper, if $\mathfrak{S}$ is an ideal of an integral domain $A$, we denote, as usual, $A:(A: \mathfrak{I})$ by $\mathfrak{\Im}_{v}$. An ideal $\mathfrak{\Im}$ is called divisorial if $\mathfrak{\Im}=\mathfrak{J}_{v}$, strong if $(A: \mathfrak{I})=(\mathfrak{I}: \mathfrak{\Im})$ (cf. [3]), strongly divisorial if it is strong and divisorial (cf. [11]).

\section{§1. The algorithmic construction}

We begin by showing that any non-zero intersection of strongly divisorial prime ideals is a strongly divisorial ideal. We need the following: 
Lemma 1.1. Let $\mathfrak{P}$ be a prime ideal containing a radical ideal $\mathfrak{S}$ of an integral domain $A$. Then $(\mathfrak{P}: \mathfrak{P}) \subset(\mathfrak{\Im}: \mathfrak{\Im})$.

Proof. Let $\mathfrak{S}=\cap\left\{\mathfrak{P}_{2} ; \lambda \in \Lambda\right\}$, where, for each $\lambda, \mathfrak{P}_{\lambda}$ is a minimal prime of $\mathfrak{J}$. Since $\mathfrak{I} \subset \mathfrak{P}$, we have $\mathfrak{I}(\mathfrak{B}: \mathfrak{\beta}) \subset \mathfrak{P}$. But, for each $\mathfrak{P}_{\lambda}$, we have $\mathfrak{I}(\mathfrak{P}: \mathfrak{P}) \subset \mathfrak{P}_{\lambda}(\mathfrak{P}: \mathfrak{P}) \subset \mathfrak{P}_{\lambda}(A: \mathfrak{P}) \subset\left(\mathfrak{P}_{\lambda}: \mathfrak{P}\right)$. Notice that, for each $\mathfrak{P}_{\lambda}$ with $\mathfrak{P}_{\lambda} \neq \mathfrak{R}$, we have $\left(\mathfrak{P}_{\lambda}: \mathfrak{P}\right) \cap A=\mathfrak{P}_{\lambda}$, because if $x \in A$ and $x \mathfrak{R} \subset \mathfrak{P}_{\lambda}$, then, since $\mathfrak{B} \not \subset \mathfrak{P}_{\lambda}, \quad x \in \mathfrak{P}_{\lambda}$. Thus we have $\mathfrak{S}(\mathfrak{P}: \mathfrak{P}) \subset \mathfrak{P} \cap\left\{\left(\mathfrak{P}_{\lambda}: \mathfrak{P}\right) ; \mathfrak{P}_{\lambda} \neq \mathfrak{P}\right\} \subset$ $\mathfrak{P} \cap\left\{\mathfrak{P}_{2} ; \mathfrak{P}_{\lambda} \neq \mathfrak{P}\right\}=\mathfrak{S}$, that is $(\mathfrak{P}: \mathfrak{P}) \subset(\mathfrak{S}: \mathfrak{S})$.

Proposition 1.2. Let $\mathfrak{S}=\cap\left\{\mathfrak{P}_{\lambda} ; \lambda \in \Lambda\right\}$, where for each $\lambda \in \Lambda$, $\mathfrak{P}_{\lambda}$ is a strongly divisorial prime ideal of an integral donxin $A$. If $\mathfrak{\Im} \neq(0)$, then $\mathfrak{\Im}$ is a strongly divisorial ideal of $A$.

Proof. It is enough to show that $\mathfrak{S}=A:(\mathfrak{S}: \mathfrak{S}$ ) (cf. [3, Proposition 6]). It is obvious that $\mathfrak{\Im} \subset A$ : ( $\mathfrak{\Im}: \mathfrak{I})$. For the opposite inclusion, since, by Lemma 1.1, $\left(\mathfrak{P}_{\lambda}: \mathfrak{P}_{\lambda}\right) \subset(\mathfrak{I}: \mathfrak{I})$ for each $\lambda \in \Lambda$, we have $\mathfrak{P}_{\lambda}=A:\left(A: \mathfrak{P}_{\imath}\right)$ $=A:\left(\mathfrak{P}_{\lambda}: \mathfrak{P}_{\lambda}\right) \supset A:(\mathfrak{J}: \mathfrak{J})$. Thus $\cap\left\{\mathfrak{P}_{\lambda} ; \lambda \in \Lambda\right\}=\mathfrak{J} \supset A:(\mathfrak{S}: \mathfrak{J})$.

For a Mori domain, a "converse" for Proposition 1.2 holds:

Proposition 1.3. Let $A$ be a Mori domain and let $\mathfrak{\Im}$ be a strongly divisorial ideal of $A$. If $\mathfrak{B}$ is a prime ideal minimal over $\mathfrak{F}$, then $\mathfrak{P}$ is strongly divisorial.

Proof. Consider the localization $A_{\mathfrak{r}}$. Since $\left(\mathfrak{S} A_{\mathfrak{\beta}}\right)_{v}=\mathfrak{J}_{v} A_{\mathfrak{p}}=\mathfrak{S} A_{\mathfrak{B}}$ and $\left(A_{\mathfrak{\beta}}: \mathfrak{S} A_{\mathfrak{\beta}}\right)=A_{\mathfrak{\beta}}(A: \mathfrak{I})=A_{\mathfrak{\beta}}(\mathfrak{S}: \mathfrak{\Im})=\left(\mathfrak{S} A_{\mathfrak{P}}: \mathfrak{S} A_{\mathfrak{\beta}}\right)$ (cf. for example [11], proof of Theorem 2), $\mathfrak{J} A_{\mathfrak{F}}$ is a strongly divisorial ideal of $A_{\mathfrak{r}}$. Therefore $\mathfrak{\Im} A_{\mathfrak{\beta}}$ is contained in at least one strong maximal divisorial ideal of $A_{\mathfrak{\beta}}$ (cf. [5, Proposition (1.7)]), that is $\mathfrak{\beta} A_{\mathfrak{\beta}}$ is strongly divisorial. By [11, Lemma 4], we conclude that $\mathfrak{\beta}$ is a strongly divisorial ideal of $A$.

As usual, we denote by $A^{*}$ the complete integral closure of $A$. We consider in the following results mainly the case where the conductor of $A$ in $A^{*},\left(A: A^{*}\right)$ is different from (0). This hypothesis is equivalent for a Noetherian domain $A$ to suppose that the integral closure of $A$, $\bar{A}=A^{*}$ is a finitely generated $A$-module.

Lemma 1.4. Let $A$ be a Mori domain such that $\left(A: A^{*}\right) \neq 0$. Then any decreasing chain of strongly divisorial ideals of $A$ is stationary.

Proof. Let $\left\{\mathfrak{\Im}_{n} ; n \geq 0\right\}$ be a strictly decreasing chain of strongly divisorial ideals of $A$. Since $A$ is a Mori domain, $\cap\left\{\mathfrak{S}_{n} ; n \geq 0\right\}=(0)$ (cf. [12, 
I, Theorem 1]). On the other hand, since $\left(A: A^{*}\right) \neq(0), \cap\left\{\widetilde{I}_{n} ; n \geq 0\right\} \neq(0)$ (cf. [3, Proposition 16]), a contradiction.

We denote, as in [4] by $D_{m}(A)$ the set of maximal divisorial ideals of a Mori domain $A$. The elements of $D_{m}(A)$ are prime ideals of $A$ and, if $\mathfrak{\beta} \in D_{m}(A)$, either $A_{\mathfrak{B}}$ is a DVR or $\mathfrak{B}$ is strong, i.e. strongly divisorial (cf. [4, Proposition (2.1) and Theorem (2.5)]). The set $\mathscr{S}(A)=\left\{\mathfrak{P} \in D_{m}(A) \mid \mathfrak{P}\right.$ is strong $\}$ is nearly related to $A^{*}$, as we shall see later. At the moment we prove:

Proposition 1.5. Let $A$ be a Mori domain such that $\left(A: A^{*}\right) \neq(0)$. Then $\mathscr{S}(A)$ is empty or finite.

Proof. The first case, $\mathscr{S}(A)=\varnothing$, occurs if and only if $A$ is a Krull domain. In fact, if $A$ is a Krull domain, it is well known that $A_{\mathfrak{B}}$ is a DVR, for each $\Re \in D_{m}(A)$ and, conversely, if $\mathscr{S}(A)=\varnothing, A$ is a Krull domain (cf. [4, Theorem (3.3)]). Suppose that $\mathscr{S}(A)$ is non empty. If $\mathscr{S}(A)$ is not finite, consider a countable set $\left\{\mathfrak{P}_{1}, \cdots \mathfrak{P}_{n}, \cdots\right\}$ of elements of $\mathscr{S}(A)$, with $\mathfrak{P}_{i} \neq \mathfrak{B}_{j}$, for $i \neq j$. We can consider the decreasing chain $\left\{\widetilde{I}_{n} ; n \geq 1\right\}$, where $\widetilde{\Im}_{n}=\bigcap\left\{\mathfrak{\beta}_{i} ; 1 \leq i \leq n\right\}$. For each $n, \widetilde{I}_{n}$ is a strongly divisorial ideal by Proposition 1.2. Moreover the chain $\left\{\widetilde{J}_{n} ; n \geq 1\right\}$ is strictly decreasing because, if $\mathfrak{\Im}_{n}=\mathfrak{\Im}_{n+1}$, then $\mathfrak{\beta}_{1} \cdots \mathfrak{P}_{n} \subset \mathfrak{\Im}_{n}=\mathfrak{\Im}_{n+1} \subset \mathfrak{\beta}_{n+1}$, thus $\mathfrak{P}_{i} \subset \mathfrak{P}_{n+1}$ for some $i, 1 \leq i \leq n$, which is clearly impossible. By Lemma 1.4 we get a contradiction.

Corollary 1.6. Let $A$ be a Mori domain such that $\left(A: A^{*}\right) \neq(0)$. Then the set of strongly divisorial prime ideals of $A$ is empty or finite.

Proof. Let $\mathscr{P}$ be the set of strongly divisorial prime ideals of $A$. $\mathscr{P}=\varnothing$ if and only if $A$ is a Krull domain (cf. [3, Corollary 14]). If $\mathscr{P} \neq \varnothing$, notice that the set of the maximal elements of $\mathscr{P}$ is exactly $\mathscr{S}(A)$. In fact, trivially, if $\mathfrak{B} \in \mathscr{S}(A), \mathfrak{P}$ is a maximal element of $\mathscr{P}$. Conversely, let $\mathfrak{R}$ be a maximal element of $\mathscr{P}$. Since $\mathfrak{R}$ is divisorial, $\mathfrak{B} \subset \mathfrak{M}$ for some $\mathfrak{M} \in D_{m}(A)$. But $\mathfrak{\beta} A_{\mathfrak{M}}$ is a strongly divisorial ideal of $A_{\mathfrak{M}}$, thus $A_{\mathfrak{M}}$ is not a $\mathrm{DVR}$ and $\mathfrak{M} \in \mathscr{S}(A) \subset \mathscr{P}$. For the maximality of $\mathfrak{B}, \mathfrak{P}=\mathfrak{M} \in \mathscr{S}(A)$. Therefore, by Proposition 1.5, the maximal elements of $\mathscr{P}$ are a finite number: $\mathfrak{B}_{1}, \cdots, \mathfrak{P}_{s}$. Arguing as in the proof of Proposition 1.5, we can show that $\mathscr{P} \backslash\left\{\mathfrak{P}_{1}, \cdots, \mathfrak{P}_{s}\right\}$ has a finite number of maximal elements $\mathfrak{P}_{1}^{\prime}, \cdots, \mathfrak{P}_{t}^{\prime}$ and trivially, for each $i, 1 \leq i \leq t, \mathfrak{P}_{i}^{\prime} \varsubsetneqq \mathfrak{P}_{j}$ for some $j, 1 \leq j$ $\leq s$. To conclude the proof it is enough to observe that any decreasing 
chain of elements of $\mathscr{P}$ is finite (cf. Lemma 1.4).

REMARK 1.7. Let $A$ be a Noetherian ring satisfying the $S_{1}$-condition and let $R, R \subset \bar{A}$, be a finite overring of $A$. In this case K. Yoshida [17] considers a sequence of intermediate rings between $A$ and $R$ (related to a sequence that we are going to introduce) and a set of distinguished prime ideals of $A, D(A, R)$ (cf. [17, Proposition-Definition 1.1]). We notice that, if $A$ is a Noetherian domain and $R=\bar{A}$, the set $D(A, \bar{A})$ of [17] coincides with the set of strongly divisorial prime ideals of $A$.

In fact, if $\Re \in \operatorname{Spec} A$ and ht $P=1$, then $\mathfrak{P} \in D(A, \bar{A})$ if only if $A_{\mathfrak{B}}$ is not integrally closed (cf. [17, p. 54]), i.e. if and only if $\Re A_{\mathfrak{B}}$ is not principal (cf. for example [1, Proposition 9.2]). It is easy to prove that the previous statement is equivalent to assume that $\mathfrak{P}$ is a strong ideal of $A$. Since in this case (ht $\mathfrak{\beta}=1) \mathfrak{B}$ is always divisorial (cf. for example [11, Proposition 1]), we have that $\mathfrak{P} \in D(A, \bar{A})$ if and only if $\mathfrak{P}$ is strongly divisorial. On the other hand, if $\mathfrak{B} \in \operatorname{Spec} A$ and ht $\mathfrak{P}>1$, then $\mathfrak{B} \in$ $D(A, \bar{A})$ if and only if $\mathfrak{\beta}$ is divisorial (cf. [17, Proposition 1.10, (vi) $\Leftrightarrow$ (xi)]). Since in this case (ht $\mathfrak{P}>1$ ) $\mathfrak{P}$ is always strong (if not $\mathfrak{\beta} A_{\mathfrak{P}}$ would be a principal ideal of the Mori domain $A_{\mathfrak{r}}$, a contradiction with [11, Lemma $3]$ ), we have that $\mathfrak{\beta} \in D(A, \bar{A})$ if and only if $\mathfrak{\beta}$ is strongly divisorial.

We notice in particular that Corollary 1.6 generalizes Yoshida's result on the finiteness of the set $\left\{\mathfrak{P} \in \operatorname{Spec} A \mid\right.$ ht $\mathfrak{\beta}>1$ and depth $\left.A_{\mathfrak{\beta}}=1\right\}$ (cf. [17, Proposition 1.10 and Corollary 1.12]).

We recall that if $A$ is a Mori domain and $\mathfrak{I}$ is a strongly divisorial ideal of $A$, then $(A: \mathfrak{I})=(\mathfrak{S}: \mathfrak{S})$ is a Mori overring of $A$ (cf. [13, p. 11] or [3, Corollary 11]). If, moreover, $A$ is a Mori domain such that $\left(A: A^{*}\right)$ $\neq(0)$, then also $(A: \mathfrak{S})$ has the same property, that is $\left((A: \mathfrak{S}):(A: \mathfrak{S})^{*}\right)$ $\neq(0)$, because $(A: \mathfrak{\Im})^{*}=A^{*}$. Thus, under the preceding hypothesis, we can construct a sequence of Mori overrings of $A$

$$
A=A_{0} \subset A_{1} \subset \cdots \subset A_{m} \subset \cdots
$$

setting for each $i \geq 0, A_{i+1}=\left(A_{i}: \mathscr{R}_{i}\right)$, where $\mathscr{R}_{i}=\cap\left\{\mathfrak{P} ; \mathfrak{P} \in \mathscr{S}\left(A_{i}\right)\right\}$, if $\mathscr{S}\left(A_{i}\right) \neq \varnothing$ and $A_{i+1}=A_{i}$, if $\mathscr{S}\left(A_{i}\right)=\varnothing$.

Notice that, in the first case, $\mathscr{R}_{i} \neq(0)$, by Proposition 1.5 , and that $\mathscr{R}_{i}$ is a strongly divisorial ideal of $A_{i}$, by Proposition 1.2 ; thus, if $\mathscr{S}\left(A_{i}\right)$ $\neq \varnothing, A_{i} \varsubsetneqq A_{i+1}$. Conversely, if $\mathscr{S}\left(A_{i}\right)=\varnothing, A_{i}=A_{j}$, for each $j \geq i$.

Theorem 1.8. Let $A$ be a Mori domain such that $\left(A: A_{*}\right) \neq(0)$. Then 
the sequence of overrings of $A$ considered above is stationary for some $m \geq 0$ and $A_{m}=A^{*}$.

Proof. For any $i, i \geq 0$ it is easy to see that $A_{i}$ is an overring of the type $\mathfrak{\Im}_{i}^{-1}$ for some ideal $\mathfrak{J}_{i}$ of $A$, that is $A_{i}$ is a (fractional) divisorial ideal of $A$. In correspondence with the sequence $\left\{A_{i} ; i \geq 0\right\}$ of overrings of $A$, we get the decreasing sequence of strongly divisorial ideals of $A$, $\left\{\left(A: A_{i}\right) ; i \geq 0\right\}$. This is stationary by Lemma 1.4, thus the sequence of overrings $\left\{A_{i} ; i \geq 0\right\}$ is stationary too (cf. [3, Corollary 8]).

Therefore there exists an $m \geq 0$ such that $A_{m}=A_{m+1}$. Thus $\mathscr{S}\left(A_{m}\right)$ $=\varnothing$ i.e. $A_{m}$ is a Krull domain (cf. [4, Theorem (3.3)]). However $A^{*}=$ $\left(A_{m}\right)^{*}$, because $\left(A: A_{m}\right) \neq(0)$ i.e. $A$ and $A_{m}$ have a nonzero ideal in common. On the other hand $A_{m}$ is completely integrally closed, that is $\left(A_{m}\right)^{*}=A_{m}$, thus $A^{*}=A_{m}$.

ExAmples 1.9. a) Let $A=k \llbracket t^{3}, t^{5} \rrbracket$, where $k$ is a field. $A$ is a 1-dimensional Noetherian (in particular Mori) local domain and its maximal ideal $\mathscr{M}=\left(t^{3}, t^{5}\right)$ is strongly divisorial. In this case $\mathscr{R}_{0}=\mathfrak{M}$ and $A_{1}=\left(A: \mathscr{R}_{0}\right)$ $=k \llbracket t^{3}, t^{5}, t^{7} \rrbracket ; \mathscr{R}_{1}=\left(t^{3}, t^{5}, t^{7}\right)$ and $A_{2}=\left(A_{1}: \mathscr{R}_{2}\right)=k \llbracket t^{2}, t^{3} \rrbracket ; \mathscr{R}_{2}=\left(t^{2}, t^{3}\right)$ and $\left.A_{3}=\left(A_{2}: \mathscr{R}_{2}\right)=k \llbracket t\right]$.

Observe that in this example our sequence of overrings of $A$ is different from the sequence constructed by J. Lipman (cf. [10, p. 661]). As a matter of fact, in this case the steps in the Lipman sequence are $k \llbracket t^{3}, t^{5} \rrbracket \subset k \llbracket t^{2}, t^{3} \rrbracket \subset k \llbracket t \rrbracket$.

b) Let $A=k+X K[X]+Y K[X, Y, Z]$, where $k \varsubsetneqq K$ are fields. $A$ is a Mori (possibly non-Noetherian) domain, because $A=K[X, Y, Z] \cap B_{1} \cap B_{2}$ where $B_{1}=k+(X, Y, Z) K[X, Y, Z]_{(X, Y, Z)}$ and $B_{2}=K(X)+Y K[X, Y, Z]_{(Y)}$ are Mori domains (cf. [12, I, Theorem 2] and [2, Proposition 3.4]). In this case $\mathscr{R}_{0}=X K[X]+Y K[X, Y, Z], \quad A_{1}=\left(A: \mathscr{R}_{0}\right)=K[X]+Y K[X, Y, Z]$, $\mathscr{R}_{1}=Y K[X, Y, Z]$ and finally $A_{2}=\left(A_{1}: \mathscr{R}_{1}\right)=K[X, Y, Z]$.

We recall that if $A$ is a domain, $\mathfrak{S}$ is a strongly divisorial ideal of $A$ and $C=(A: \mathfrak{\Im})$, then Spec $A$ and Spec $C$ are closely related. More precisely the canonical map associated to the inclusion $i: A \rightarrow C,{ }^{a} i$ : Spec $C$ $\rightarrow$ Spec $A$ gives a one-to-one correspondence between $\{\mathfrak{Q} \in \operatorname{Spec} C \mid \mathfrak{D} \not \supset \mathfrak{I}\}$ and $\{\mathfrak{P} \in \operatorname{Spec} A \mid \mathfrak{P} \not \supset \mathfrak{I}\}$; moreover, if $\mathfrak{Q} \in \operatorname{Spec} C, \mathfrak{Q} \not \supset \mathfrak{I}$ and $\mathfrak{P}=\mathfrak{Q} \cap A$, then $C_{\mathfrak{Q}}=A_{\mathfrak{\beta}}$ (cf. for instance [7, Theorem 1.4, c)]). We notice also that for any $\mathfrak{P} \in \operatorname{Spec} A, \mathfrak{P} \not \supset \mathfrak{J}$, the unique $\mathfrak{D} \in \operatorname{Spec} C$ above $\mathfrak{P}$ is ( $\mathfrak{P}: \mathfrak{S}$ ). Actually ( $\mathfrak{P}: \mathfrak{I})$ is a prime ideal of $C$, because if $a b \in(\mathfrak{P}: \mathfrak{S})$ and $a \notin(\mathfrak{P}: \mathfrak{S})$, 
with $a, b \in C=(A: \mathfrak{I})$, then $a b \in\left(\mathfrak{B}: \mathfrak{J}^{2}\right)$ i.e. $a \mathfrak{J} b \mathfrak{I} \subset \mathfrak{P}$, so, since $a \mathfrak{S} \subset A$, $b \mathfrak{I} \subset A$ and $a \mathfrak{s} \not \subset \mathfrak{P}$, we have $b \mathfrak{S} \subset \mathfrak{P}$, that is $b \in(\mathfrak{P}: \mathfrak{J})$. Moreover $(\mathfrak{P}: \mathfrak{I}) \cap A=\mathfrak{B}$, because if $x \in A$ is such that $x \mathfrak{S} \subset \mathfrak{B}$, then, since $\mathfrak{I} \not \subset \mathfrak{B}$, $x \in \mathfrak{P}$, and, on the other hand, it is trivial that $\mathfrak{P} \subset(\mathfrak{P}: \mathfrak{I}) \cap A$.

We want to show that, if $A$ is a Mori domain, in the previous oneto-one correspondence, strongly divisorial primes of $C$ correspond to strongly divisorial primes of $A$.

Proposition 1.10. Let $A$ be a Mori domain, $\mathfrak{\Im}$ a strongly divisorial ideal of $A$ and $C=(A: \mathfrak{I})$. If $\mathfrak{\beta} \in \operatorname{Spec} A, \mathfrak{\beta} \not \supset \mathfrak{\Im}$ and $\mathfrak{Q}=(\mathfrak{B}: \mathfrak{\Im})$ (i.e. $\mathfrak{\cap} A=\mathfrak{P})$, then $\mathfrak{P}$ is a strongly divisorial ideal of $A$ if and only if $\mathfrak{Q}$ is a strongly divisorial ideal of $C$. Moreover if $\mathfrak{B} \in \mathscr{S}(A)$, then $\mathfrak{Q} \in \mathscr{S}(C)$.

Proof. We know that $C$ is a Mori domain and that, if $\Re \in \operatorname{Spec} A$, $\mathfrak{P} \not \supset \mathfrak{I}$, is a strongly divisorial ideal of $A$, then $\mathfrak{D}=(\mathfrak{B}: \mathfrak{S})$ is a divisorial ideal of $C$ (cf. [13, p. 11]). We want to prove that $\mathfrak{Q}$ is strong.

Denote by $F$ the quotient field of $A$ (and of $C$ ). If $\mathfrak{Q}$ is not strong,

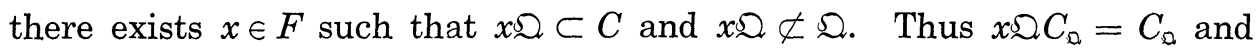
$\mathfrak{a} C_{\mathfrak{Q}}=x^{-1} C_{\mathfrak{Q}}$ is principal. But $C_{\mathfrak{Q}}$ is a Mori domain (cf. [11, Corollary 3]) and so if ht $\mathfrak{Q} \geq 2$, we have a contradiction with [11, Lemma 2]. On the other hand, if ht $\mathfrak{Q}=1, C_{\mathfrak{Q}}=A_{\mathfrak{B}}$ is a DVR (cf. [13, Theorem A-4]). This also is a contradiction because $\mathfrak{B}$ (and consequently $\mathfrak{P} A_{\mathfrak{R}}$ ) is strong.

Conversely, let $\mathfrak{Q}=(\mathfrak{B}: \mathfrak{S})$ be a strongly divisorial ideal of $C$, with $\mathfrak{P} \in \operatorname{Spec} A, \mathfrak{B} \not \supset \mathfrak{I}$. As noted before, $\mathfrak{P}=\mathfrak{Q} \cap A$, thus it is easy to see that $\mathfrak{P}$ is a divisorial ideal of $A$. In fact, since $\mathfrak{Q}=\cap\{x C ; x \in F$ and $x C \supset \mathfrak{Q}\}, \mathfrak{P}=\cap\{x(A: \mathfrak{\Im}) ; x \in F$ and $x C \supset \mathfrak{Q}\} \cap A$ is an intersection of divisorial ideals of $A$. We want to prove now that $\mathfrak{B}$ is strong, i.e. that $(A: \mathfrak{P})=(\mathfrak{P}: \mathfrak{P})$. Actually we have $(A: \mathfrak{P}) \subset(A: \mathfrak{I} \mathfrak{Q})=((A: \mathfrak{S}): \mathfrak{Q})=$ $(C: \mathfrak{Q})=(\mathfrak{Q}: \mathfrak{Q})$. Thus if $x \in(A: \mathfrak{P}), x \mathfrak{R} \subset x \mathfrak{Q} \subset \mathfrak{Q}$. From $x \mathfrak{R} \subset A$ and $x \mathfrak{P} \subset \mathfrak{Q}$, we get $x \mathfrak{P} \subset A \cap \mathfrak{Q}=\mathfrak{P}$, so $x \in(\mathfrak{P}: \mathfrak{P})$.

For the last part of Proposition notice that if $\mathfrak{\beta} \in D_{m}(A)$ and $\mathfrak{Q}=(\mathfrak{B}: \mathfrak{S})$ $\subset \mathfrak{M} \in D_{m}(C)$, then $\mathfrak{M} \cap A$ is a divisorial ideal of $A$. Thus $\mathfrak{M} \cap A=\mathfrak{P}$ and, for the one-to-one correspondence, $\mathfrak{Q}=\mathfrak{M}$.

Given a Mori domain $A$ such that $\left(A: A^{*}\right) \neq(0)$, we have associated to $A$ a sequence of Mori overrings:

$$
A=A_{0} \varsubsetneqq A_{1} \varsubsetneqq \cdots \varsubsetneqq A_{m}=A^{*} .
$$

From the previous Proposition we get the following: 
Corollary 1.11. Let $A$ be a Mori domain such that $\left(A: A^{*}\right) \neq(0)$ and let $\left(^{*}\right)$ be the associated sequence. Then $m \geq \sup \{$ lengths of chains of strongly divisorial primes of $A\}$.

Proof. Let $l_{i}=\sup$ \{lengths of chains of strongly divisorial primes of $\left.A_{i}\right\}$ and let $\mathfrak{P}_{0} \subset \mathfrak{P}_{1} \subset \cdots \subset \mathfrak{P}_{l_{i}}$ be a chain of strongly divisorial primes of $A_{i}$. Then necessarily $\mathfrak{P}_{l_{i}} \in \mathscr{S}\left(A_{i}\right)$ and $\mathfrak{P}_{0}, \cdots, \mathfrak{P}_{l_{i-1}} \not \supset \mathscr{R}_{i}=\cap\left\{\mathfrak{P} ; \mathfrak{P} \in \mathscr{S}\left(A_{i}\right)\right\}$. So, by Proposition 1.10, there exists in $A_{i+1}=\left(A_{i}: \mathscr{R}_{i}\right)$ a chain of strongly divisorial primes of length at least $l_{i}-1$. Recalling that $A_{m}$ is the only ring in the sequence $(*)$ which does not have strongly divisorial primes, the conclusion follows easily.

Other informations about the relationship between strongly divisorial primes of two consecutive rings of the sequence $\left(^{*}\right)$ are given in the following:

Proposition 1.12. Let $A$ be a Mori domain such that $\left(A: A^{*}\right) \neq(0)$ and let $B, C=(B: \mathscr{R})$ be consecutive (Mori) domains of the associated sequence $\left.{ }^{*}\right)$, where $\mathscr{R}=\mathfrak{P}_{1} \cap \cdots \cap \mathfrak{P}_{n}$ and $\left\{\mathfrak{P}_{1}, \cdots, \mathfrak{P}_{n}\right\}=\mathscr{S}(B)$. If $\mathfrak{Q}$ is a strongly divisorial prime ideal of $C$ such that $\mathfrak{Q} \supset \mathscr{R}$, then $\mathfrak{Q} \cap B=\mathfrak{P}_{j}$ for some $j, j=1, \cdots, n$.

Proof. As in the proof of Proposition 1.10 it is easy to see that $\mathfrak{\cap} B$ is a divisorial ideal of $B$. But, since $\mathfrak{Q} \mathscr{R}$ and $B \supset \mathscr{R}, \mathfrak{P}=\mathfrak{Q} \cap B$ $\supset \mathscr{R}=\mathfrak{P}_{1} \cap \cdots \cap \mathfrak{P}_{n} \supset \mathfrak{P}_{1} \cdots \mathfrak{P}_{n}$. Since $\mathfrak{R}$ is a prime ideal, $\mathfrak{R} \supset \mathfrak{P}_{j}$ for some $j, j=1, \cdots, n$. Thus $\mathfrak{R}=\mathfrak{P}_{j}$, becasue $\mathfrak{\beta}$ is divisorial and $\mathfrak{R}_{j}$ is maximal divisorial in $B$.

For an example of the situation described in Proposition 1.12, look at Example 1.9 a). $A_{1}$ (resp. $A_{2}$ ) has a strongly divisorial prime, $\mathscr{R}_{1}$ (resp. $\mathscr{R}_{2}$ ), above $\mathscr{R}_{0} \in \mathscr{S}(A)$ (resp. $\mathscr{R}_{1} \in \mathscr{S}\left(A_{1}\right)$ ).

Clearly in this case, if (*) is the associated sequence of overrings of $A, m>\sup \{$ lengths of chains of strongly divisorial primes of $A$ \}.

Proposition 1.13. Let $A$ be a Mori domain and let $\mathfrak{P}_{1}, \cdots, \mathfrak{P}_{n} \in \mathscr{S}(A)$. If $\mathscr{R}=\mathfrak{P}_{1} \cap \cdots \cap \mathfrak{P}_{n}$ and $C=(A: \mathscr{R})$, then $A=C \cap A_{\mathfrak{P}_{1}} \cap \cdots \cap A_{\mathfrak{P}_{n}}$.

Proof. The inclusion $A \subset C \cap A_{\mathfrak{P}_{1}} \cap \cdots \cap A_{\mathfrak{P}_{n}}$ is trivial. For the opposite inclusion we recall that if $A$ is a Mori domain, $A=\cap\left\{A_{\mathfrak{\beta}} ; \Re \in D_{m}(A)\right\}$ (cf. [4, Proposition (2.2) b)]). Thus it is enough to show that $C \subset A_{\mathfrak{*}}$, for any maximal divisorial ideal $\mathfrak{P}$ of $A, \mathfrak{P} \neq \mathfrak{P}_{1}, \cdots, \mathfrak{P}_{n}$. Actually for such 
maximal divisorial ideal $\mathfrak{P}$ of $A, \mathfrak{B} \not \supset \mathscr{R}=\mathfrak{B}_{1} \cap \cdots \cap \mathfrak{P}_{n}$, thus there is exactly one $\mathfrak{Q} \in$ Spec $C$ above $\mathfrak{B}$ and $A_{\mathfrak{B}}=C_{\mathfrak{Q}}$ (cf. [7, Theorem 1.4, c)]). Therefore it is clear that $C \subset A_{\mathfrak{P}}$.

Next we study in greater detail the generic step $A_{i} \subset A_{i+1}$ in the sequence (*). Putting $A_{i}=B$ and $A_{i+1}=C$ and using the notation of Proposition 1.12, we describe the extension $B \subset C$ in $n$ steps, in correspondence with the $n$ prime ideals $\mathfrak{\beta}_{1}, \cdots, \mathfrak{\beta}_{n}$.

We shall denote by $\mathscr{D}(A)$ the set of divisorial ideals of a domain $A$.

Let $B_{0}=B$ and $\alpha_{0}: \mathscr{D}(B) \rightarrow \mathscr{D}(B)$ the identity map. Define, for $1 \leq j$ $\leq n$, the pair $\left(B_{j}, \alpha_{j}\right)$ in the following way:

$$
\begin{aligned}
& B_{j}=B_{j-1}:\left(\alpha_{j-1} \circ \cdots \circ \alpha_{0}\left(\mathfrak{P}_{j}\right)\right) \\
& \alpha_{j}: \mathscr{D}\left(B_{j-1}\right) \longrightarrow \mathscr{D}\left(B_{j}\right) \\
& H \longrightarrow H:\left(\alpha_{j-1} \circ \cdots \circ \alpha_{0}\left(\mathfrak{P}_{j}\right)\right)
\end{aligned}
$$

Denote, for simplicity, the map $\left(\alpha_{j-1} \circ \cdots \circ \alpha_{0}\right): \mathscr{D}(B) \rightarrow \mathscr{D}\left(B_{j-1}\right)$ by $\Psi_{j-1}$.

Observe that, for each $j, j=1, \cdots, n, \Psi_{j-1}\left(\mathfrak{P}_{j}\right) \in \mathscr{S}\left(B_{j-1}\right)$. In fact, if $j=1, \Psi_{0}\left(\mathfrak{B}_{1}\right)=\mathfrak{\beta}_{1} \in \mathscr{S}\left(B_{0}\right)$. If $j \geq 2$, applying Proposition 1.10 , we get that $\Psi_{k}\left(\mathfrak{P}_{j}\right) \in \mathscr{S}\left(B_{k}\right)$ and $\Psi_{k}\left(\mathfrak{P}_{j}\right) \not \supset \Psi_{k}\left(\mathfrak{P}_{k+1}\right)$ for any $k, k=0,1, \cdots, j-2$. So, again by Proposition 1.10, $\Psi_{j-1}\left(\Re_{j}\right) \in \mathscr{S}\left(B_{j-1}\right)$.

Therefore we have a sequence of Mori overrings of $B, B=B_{0} \subset B_{1}$ $\subset \ldots \subset B_{n}$ (cf. again [13, p. 11]). We can prove:

Proposition 1.14. Preserving the notation introduced above, the integral domain $B_{n}$ coincides with $C$.

Proof. Observe first that for each $j, j=1, \cdots, n, \Psi_{j-1}\left(\mathfrak{P}_{j}\right)$ is a fractional ideal of $B$ and that

$$
\begin{aligned}
B_{n} & =\left(B_{n-1}: \Psi_{n-1}\left(\mathfrak{P}_{n}\right)\right)=\left(B_{n-2}: \Psi_{n-2}\left(\mathfrak{P}_{n-1}\right)\right):\left(\Psi_{n-1}\left(\mathfrak{P}_{n}\right)\right) \\
& =B_{n-2}:\left(\Psi_{n-2}\left(\mathfrak{P}_{n-1}\right) \Psi_{n-1}\left(\mathfrak{P}_{n}\right)\right)=\cdots=B:\left(\Psi_{0}\left(\mathfrak{P}_{1}\right) \cdots \Psi_{n-1}\left(\mathfrak{P}_{n}\right)\right) .
\end{aligned}
$$

Observe secondly that, since for each $j, j=1, \cdots, n, \mathfrak{P}_{j} B_{\mathfrak{P}_{j}}=\left(\mathfrak{P}_{j} B_{\mathfrak{P}_{j}}\right)_{v}$ $=\left(\mathfrak{P}_{1} \cdots \mathfrak{P}_{n} B_{\mathfrak{P}_{j}}\right)_{v}$, we have $\mathfrak{P}_{1} \cap \cdots \cap \mathfrak{P}_{n}=\mathfrak{P}_{1} B_{\mathfrak{P}_{1}} \cap \cdots \cap \mathfrak{P}_{n} B_{\mathfrak{P}_{n}} \cap B=\mathfrak{P}_{1} B_{\mathfrak{\beta}_{1}} \cap$ $\cdots \cap \mathfrak{P}_{n} B_{\mathfrak{P}_{n}} \cap\left\{B_{\mathfrak{P}} ; \mathfrak{P} \in D_{m}(B), \mathfrak{P} \neq \mathfrak{P}_{j}\right\}=\left(\mathfrak{P}_{1} \cdots \mathfrak{P}_{n} B_{\mathfrak{P}_{1}}\right)_{v} \cap \cdots \cap\left(\mathfrak{P}_{1} \cdots \mathfrak{P}_{n} B_{\mathfrak{P}_{n}}\right)_{v}$ $\cap\left\{\left(\mathfrak{P}_{1} \ldots \mathfrak{P}_{n} B_{\mathfrak{\beta}}\right)_{v} ; \mathfrak{P} \in D_{m}(B), \mathfrak{P} \neq \mathfrak{P}_{j}\right\}=\left(\mathfrak{P}_{1} \ldots \mathfrak{P}_{n}\right)_{v}$ (cf. [4, Proposition (2.2), c)]).

Thus we have $C=\left(B: \mathfrak{P}_{1} \cap \cdots \cap \mathfrak{P}_{n}\right)=\left(B:\left(\mathfrak{P}_{1} \cdots \mathfrak{P}_{n}\right)_{v}\right)=\left(B: \mathfrak{P}_{1} \cdots \mathfrak{P}_{n}\right)$. Now, since for each $j, j=1, \cdots, n, \mathfrak{P}_{j} \subset \Psi_{j-1}\left(\mathfrak{P}_{j}\right)$, we have $\mathfrak{P}_{1} \cdots \mathfrak{P}_{n} \subset \Psi_{0}\left(\mathfrak{P}_{1}\right)$ $\ldots \Psi_{n-1}\left(\mathfrak{P}_{n}\right)$ and so $C \supset B_{n}$. For the opposite inclusion it is enough to 
show by induction that $\Psi_{0}\left(\mathfrak{P}_{1}\right) \ldots \Psi_{n-1}\left(\mathfrak{P}_{n}\right) \subset \mathfrak{P}_{1} \cap \cdots \cap \mathfrak{P}_{n}$. Trivially $\Psi_{0}\left(\mathfrak{P}_{1}\right)$ $=\mathfrak{P}_{1} \subset \mathfrak{P}_{1}$. Suppose that $\Psi_{0}\left(\mathfrak{P}_{1}\right) \cdots \Psi_{n-2}\left(\mathfrak{P}_{n-1}\right) \subset \mathfrak{P}_{1} \cap \cdots \cap \mathfrak{B}_{n-1} \quad(n \geq 2)$. Since $\Psi_{n-1}\left(\mathfrak{B}_{n}\right) \subset B_{n-1}$ and $\Psi_{n-2}\left(\mathfrak{B}_{n-1}\right)$ is an ideal of $B_{n-1}$, we have that $\Psi_{n-2}\left(\mathfrak{P}_{n-1}\right) \Psi_{n-1}\left(\mathfrak{P}_{n}\right) \subset \Psi_{n-2}\left(\mathfrak{P}_{n-1}\right)$, thus $\Psi_{0}\left(\mathfrak{P}_{1}\right) \cdots \Psi_{n-1}\left(\mathfrak{P}_{n}\right) \subset \mathfrak{P}_{1} \cap \cdots \cap \mathfrak{P}_{n-1}$.

Moreover, since by definition $\Psi_{n-1}\left(\mathfrak{P}_{n}\right)=\left(\Psi_{n-2}\left(\mathfrak{P}_{n}\right): \Psi_{n-2}\left(\mathfrak{P}_{n-1}\right)\right)$, it is clear that $\Psi_{n-1}\left(\mathfrak{P}_{n}\right) \Psi_{n-2}\left(\mathfrak{P}_{n-1}\right) \subset \Psi_{n-2}\left(\mathfrak{\beta}_{n}\right)$. So $\Psi_{0}\left(\mathfrak{P}_{1}\right) \ldots \Psi_{n-1}\left(\mathfrak{P}_{n}\right) \subset \Psi_{n-2}\left(\mathfrak{P}_{n}\right) \cap$ $B=\mathfrak{\beta}_{n}$ and $\Psi_{0}\left(\mathfrak{P}_{1}\right) \ldots \Psi_{n-1}\left(\mathfrak{P}_{n}\right) \subset \mathfrak{P}_{1} \cap \cdots \cap \mathfrak{P}_{n-1} \cap \mathfrak{P}_{n}$.

\section{§2. Contraction of ideals and glueings}

To descend in the sequence $\left(^{*}\right)$ associated to a Mori domain, defined in Section 1, we need some further definitions.

Definition 2.1. Let $A \subset B$ be two rings and let $\mathfrak{S}$ be an integral ideal of $B$ such that $\mathfrak{I} \cap A=\mathfrak{p} \in \operatorname{Spec} A$. Let $S=A \mid \mathfrak{p} . \quad S$ is a multiplicative set of $A$ and of $B$. Denote by $\phi$ the composition of canonical maps $B \rightarrow S^{-1} B \rightarrow S^{-1} B / S^{-1} \mathfrak{S}$ and by $k(\mathfrak{p})$ the residue field $A_{\mathfrak{p}} / \mathfrak{p} A_{\mathfrak{p}}$. Let $k(\mathfrak{p}) \rightarrow S^{-1} B / S^{-1} \mathfrak{S}$ be the canonical immersion. Then the ring obtained from $B$ by contracting $\mathfrak{J}$ over $\mathfrak{p}$ is the pullback $\phi^{-1}(k(\mathfrak{p}))=B \times_{S^{-1 B / S-1 \mathfrak{Y}}} k(\mathfrak{p})$.

Remark 2.2. a) In Definition 2.1, if $\mathfrak{S}$ is an intersection of a family $\left\{\mathfrak{Q}_{\lambda} ; \lambda \in \Lambda\right\}$ of primary ideals of $B$, such that $\mathfrak{Q}_{\lambda} \cap A=\mathfrak{p}$, for each $\lambda \in \Lambda$, then the ring obtained from $B$ by contracting $\mathfrak{J}$ over $\mathfrak{p}$ coincides with the ring obtained from $B$ by glueing the primary ideals $\left\{\mathfrak{Q}_{\lambda} ; \lambda \in \Lambda\right\}$ over $\mathfrak{p}$, as defined in [14] (cf. [14, Proposition 1.5]).

b) If we suppose that $\mathfrak{S}=\sqrt{\mathfrak{p} B}$, that is if $\mathfrak{\Im}$ is an intersection of a family $\left\{\mathfrak{P}_{\lambda} ; \lambda \in A\right\}$ of prime ideals of $B$, then the ring obtained from $B$ by contracting $\mathfrak{S}$ over $\mathfrak{p}$, defined in 2.1 , coincides with the ring obtained from $B$ by glueing over $\mathfrak{p}$, as defined in [9]. In particular, if $B$ is integral and finite over $A$ (and $\mathfrak{S}=\sqrt{\mathfrak{p} B}$ ), then the family $\left\{\mathfrak{P}_{\lambda} ; \lambda \in \Lambda\right\}$ is finite and, locally, for each $\lambda, S^{-1} \mathfrak{P}_{\lambda}$ is a maximal ideal of $S^{-1} B$. Thus, in this case, the pullback diagram is of the following form:

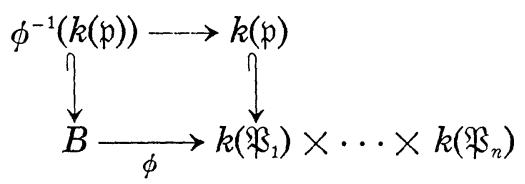

and we obtain the "classical" definition of the ring obtained from $B$ by glueing over $\mathfrak{p}$, as defined in [16]. 
c) Notice that to define properly the ring obtained from $B$ by glueing over $\mathfrak{p} \in \operatorname{Spec} A$ (i.e. by contracting $\mathfrak{J}=\sqrt{\mathfrak{p} B}$ over $\mathfrak{p}$ ) or the ring obtained from $B$ by contracting $\mathfrak{S}=\mathfrak{p} B$ over $\mathfrak{p}$, it is necessary that one of the following equivalent conditions holds: tion);

i) the canonical map $A / p \rightarrow B / p B$ is injective (cf. Iscebeck's defini-

ii) $\mathfrak{p} B$ is over $\mathfrak{p}$, that is $\mathfrak{p} B \cap A=\mathfrak{p}$;

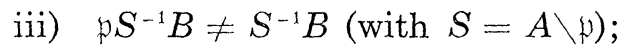

iv) there exists a prime ideal $\mathfrak{a}$ of $B$ over $\mathfrak{p}$;

v) $\sqrt{p B}$ is over $p$.

Using the hypotheses and notation of Definition 2.1, we can show that:

Prcposition 2.3. The ring obtained from $B$ by contracting $\mathfrak{J}$ over $p$ is the largest subring $A^{\prime}$ of $B$ such that

i) $\mathfrak{S}=\mathfrak{p}^{\prime}$ is a prime ideal of $A^{\prime}$;

ii) the canonical homomorphism $k(p) \rightarrow k\left(p^{\prime}\right)$ is an isomorphism.

Proof. Notice that in our hypotheses, we have the following commutative diagram:

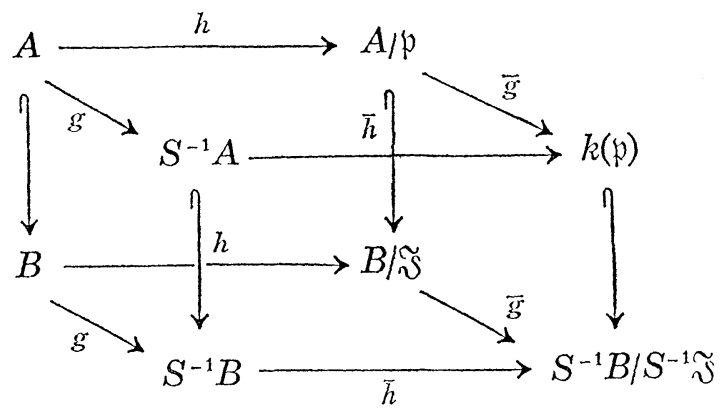

Observe moreover that $S^{-1} B / S^{-1} \mathfrak{S}=\bar{S}^{-1}(B / \mathfrak{I})$, where $\bar{S}=h(S)=$ $\{s+\mathfrak{J} ; s \in S\}$ is a multiplicative part of $B / \mathfrak{J}$. Since in $\bar{S}$ there are not zero-divisors (in fact $\left(s_{1}+\mathfrak{J}\right)\left(s_{2}+\mathfrak{S}\right)=\mathfrak{J}$, with $s_{1}, s_{2} \in S$, implies $s_{1} s_{2} \in \mathfrak{p}$ and so $s_{1} \in \mathfrak{p}\left(\right.$ and $\left.\left(s_{1}+\mathfrak{J}\right)=\mathfrak{J}\right)$ or $s_{2} \in \mathfrak{p}\left(\right.$ and $\left.\left.\left(s_{2}+\mathfrak{J}\right)=\mathfrak{J}\right)\right)$ the homomorphism $\bar{g}$ is injective.

Let $C$ be the ring obtained from $B$ by contracting $\mathfrak{\Im}$ over $\mathfrak{p}$. By definition $C=\phi^{-}(k(p))$, where $\phi=\bar{h} \circ g=\bar{g} \circ h$. Thus, considering the injection $\bar{g}$ as an inclusion, $C$ is the pullback of the diagram 


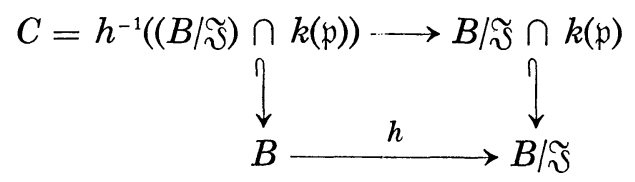

where the intersection is in $S^{-1} B / S^{-1} \mathfrak{J}$.

Since $C / \mathfrak{S}=B / \mathfrak{S} \cap k(\mathfrak{p})$ is an integral domain, $\mathfrak{S}=\mathfrak{p}^{\prime}$ is a prime ideal of $C$. Therefore $C$ is a ring that contains $A$ and has a prime ideal $\mathfrak{p}^{\prime}$ over $\mathfrak{p}$ and hence we have the canonical monomorphism $k(\mathfrak{p}) \rightarrow k\left(\mathfrak{p}^{\prime}\right)$. However $k\left(\mathfrak{p}^{\prime}\right)$ is the quotient field of $C / \mathfrak{p}^{\prime}=B / \mathfrak{S} \cap k(\mathfrak{p})$, thus it is contained in $k(\mathfrak{p})$ and so $k(\mathfrak{p}) \cong k\left(\mathfrak{p}^{\prime}\right)$.

Now, we want to show that $C$ is maximal with respect to the properties i) and ii). A subring of $B$ with properties i) and ii) is in fact a pullback of the type $B \times_{B / \mathcal{S}} D$ where $D$ is a domain contained in $B / \mathfrak{S}$ and containing $A / \mathfrak{p}$ and with quotient field isomorphic to $k(\mathfrak{p})$. The largest ring of this kind is clearly $C$, constructed in correspondence with the largest $D=B / \mathfrak{S} \cap k(\mathfrak{p})$ with the described properties.

Remark 2.4. Observe that if $C$ is the ring obtained from $B$ by contracting $\mathfrak{S}$ over $\mathfrak{p} \in \operatorname{Spec} A$, then:

a) $C$ may have also other primes over $\mathfrak{p}$ (cf. [14, Oss. 1, p. 5]).

b) $A+\mathfrak{S} \subset C$ and, with an analogous argument to [14, Proposition 1.7], it can be shown that $A+\mathfrak{S}=C$ if and only if $A / \mathfrak{p}=C / \mathfrak{S}(=B / \mathfrak{S} \cap$ $k(p))$.

The following example shows that it may be $A \varsubsetneqq A+\mathfrak{J} C$.

Example 2.5. Let $A=D+Z K[Z]$, where $D$ is a domain, $K$ its quotient field. Let $B=K[Y, Z]$ and $\mathfrak{S}=Z K[Y, Z]$. Clearly $\mathfrak{\Im} \cap A=\mathfrak{p}=$ $Z K[Z]$. In this case the ring obtained from $B$ by contracting $\mathfrak{S}$ over $\mathfrak{p}$ is the pullback of the diagram:

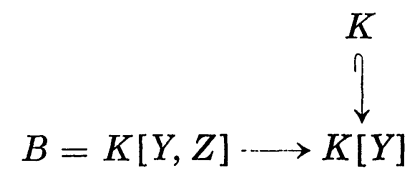

Thus it is $C=K+Z K[Y, Z]$ and $A=D+Z K[Z] \varsubsetneqq A+\mathfrak{\Im}=D+$ $Z K[Y, Z] \varsubsetneqq C$.

We extend Definition 2.1 to finitely many prime ideals:

Definition 2.6. Let $A \subset B$ be two rings and let $\mathfrak{I}_{1}, \cdots, \mathfrak{S}_{n}$ be integral ideals of $B$ such that $\mathfrak{I}_{j} \cap A=\mathfrak{p}_{j} \in \operatorname{Spec} A, j=1, \cdots, n$. We call 
the ring $B_{1} \cap \cdots \cap B_{n}$ the ring obtained from $B$ by contracting $\widetilde{J}_{1}$ over $\mathfrak{p}_{1}, \cdots, \mathfrak{\Im}_{n}$ over $\mathfrak{p}_{n}$, where for each $j, j=1, \cdots, n, B_{j}$ is the ring obtained from $B$ by contracting $\mathfrak{S}_{j}$ over $\mathfrak{p}_{j}$.

Proposition 2.7. Let $A$ be a Mori domain and let $\mathfrak{\beta}_{1}, \cdots, \mathfrak{P}_{n} \in \mathscr{S}(A)$. If $\mathscr{R}=\mathfrak{P}_{1} \cap \cdots \cap \mathfrak{P}_{n}$ and $C=(A: \mathscr{R})$, then $A$ is the ring obtained from $C$ by contracting $\mathfrak{P}_{1} C$ over $\mathfrak{P}_{1}, \mathfrak{P}_{2} C$ over $\mathfrak{P}_{2}, \cdots, \mathfrak{P}_{n} C$ over $\mathfrak{P}_{n}$.

Proof. By Proposition 1.13, we have $A=C \cap A_{\Re_{1}} \cap \cdots \cap A_{\mathfrak{P}_{n}}$. Thus it is enough to show that for each $j, j=1, \cdots, n, C \cap A_{\Re_{j}}$ is the ring obtained from $C$ by contracting $\mathfrak{P}_{j} C$ over $\mathfrak{P}_{j}$. If $S_{j}=A \backslash \mathfrak{P}_{j}$ first observe that $S_{j}^{-1} C=S_{j}^{-1}\left(A: \mathfrak{P}_{1} \cap \cdots \cap \mathfrak{P}_{n}\right)=\left(S_{j}^{-1} A:\left(S_{j}^{-1} \mathfrak{\beta}_{1} \cap \cdots \cap S_{j}^{-1} \mathfrak{\beta}_{j} \cap \cdots \cap S_{j}^{-1} \mathfrak{P}_{n}\right)\right)$ (cf. for example [11, proof of Theorem 2] for the first equality and [1, Proposition $3.11 \mathrm{v})$,] for the second). Thus $S_{j}^{-1} C=\left(S_{j}^{-1} A: S_{j}^{-1} \Re_{j}\right)=$ $S_{j}^{-1}\left(A: \mathfrak{P}_{j}\right)$. Using this equality, it is not difficult to see that the following diagram

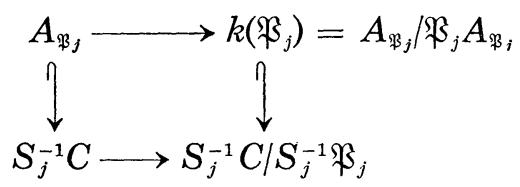

is a pullback. Recalling now that $C$ is a domain and so the canonical map $g: C \rightarrow S_{j}^{-1} C$ is injective, we can see that $C \cap A_{\mathfrak{B}_{j}}$ coincides with the pullback of the diagram

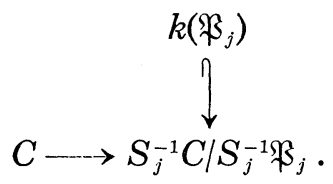

That is, $C \cap A_{\mathfrak{P}_{j}}$ is the ring obtained from $C$ contracting $\mathfrak{P}_{j} C$ over $\mathfrak{P}_{j}$.

Corollary 2.8. Let $A$ be a Mori domain such that $\left(A: A^{*}\right) \neq(0)$ and let $B, C=(B: \mathscr{R})$ be two consecutive (Mori) domains of the associated sequence $\left(^{*}\right)$ of Section 1 , where $\mathscr{R}=\mathfrak{B}_{1} \cap \cdots \cap \mathfrak{P}_{n}$ and $\mathfrak{P}_{1}, \cdots, \mathfrak{P}_{n}$ are the strong maximal divisorial ideals of $B$. Then $B$ is exactly the ring obtained from $C$ by contracting $\mathfrak{P}_{1} C$ over $\mathfrak{B}_{1}, \mathfrak{P}_{2} C$ over $\mathfrak{R}_{2}, \cdots, \mathfrak{P}_{n} C$ over $\mathfrak{\beta}_{n}$.

\section{§3. The "seminormal" case}

Let $A$ be a Mori domain such that $\left(A: A^{*}\right) \neq(0)$. Let

$$
A=A_{0} \varsubsetneqq A \varsubsetneqq \cdots \varsubsetneqq A_{m}=A^{*}
$$


be the sequence of overrings of $A$ constructed in Section 1 .

Section 3 is devoted to study the particular case where $\mathscr{R}_{i}=$ $\left(A_{i}: A_{i+1}\right)$ is a radical ideal of $A_{i+1}$, for each $i, i=0, \cdots, m-1$. As we shall see, this case is closely related to Traverso's seminormalization.

It is convenient to define the strong dimension of an integral domain $A, \operatorname{dim}_{s} A$, to be the supremum of the lengths of all chains of strongly divisorial prime ideals in $A$. If $A$ contains no proper strongly divisorial prime ideal, we say that $A$ has strong dimension -1 ; thus, if $A$ is completely integrally closed, then $\operatorname{dim}_{s} A=-1$ (cf. for example [3, Corollary 13]).

In our hypothesis, by Corollary $1.6, \operatorname{dim}_{s} A$ is finite and, by [3, Corollary 14], $A$ is completely integrally closed if and only if $\operatorname{dim}_{s} A=-1$.

LEMMA 3.1. Let $\mathfrak{\Im}$ be a strongly divisorial ideal of a domain $A$ and let $B=(A: \mathfrak{S})$. If $\mathfrak{I}$ is a radical ideal of $B$ and if $\mathfrak{\Im} \subset \mathfrak{D} \in \operatorname{Spec} B$, then $\mathfrak{Q}$ is not a strongly divisorial ideal of $B$.

Proof. Let $\mathfrak{I} \subset \mathfrak{Q} \in \operatorname{Spec} B$. Restrict $\mathfrak{Q}$ to a minimal prime $\mathfrak{P}$ of $\mathfrak{J}$. By Lemma $1.1(\mathfrak{P}: \mathfrak{P}) \subset(\mathfrak{S}: \mathfrak{S})$ and, by [8, Lemma 3.7] $(\mathfrak{Q}: \mathfrak{Q}) \subset(\mathfrak{P}: \mathfrak{P})$. Since $(\mathfrak{I}: \mathfrak{I})=(A: \mathfrak{I})=B$, we have $(\mathfrak{Q}: \mathfrak{l})=B$. If $\mathfrak{O}$ is strong, then $(B: \mathfrak{Q})=(\mathfrak{Q}: \mathfrak{Q})=B$ and $\mathfrak{l}_{v}=B$, thus $\mathfrak{Q}$ is not divisorial.

Proposition 3.2. Let $A$ be a Mori domain such that $\left(A: A^{*}\right) \neq(0)$ and let $\left(^{*}\right)$ be the associated sequence. If, for each $i, i=0, \cdots, m-1$, $\mathscr{R}_{i}=\left(A_{i}: A_{i+1}\right)$ is a radical ideal of $A_{i+1}$, then:

1) no strongly divisorial prime ideal of $A_{i+1}$ contains $\mathscr{R}_{i}$, for each $i$, $i=0, \cdots, m-1$;

2) $\operatorname{dim}_{s} A_{i}=m-i-1$, for each $i, i=0, \cdots, m$. In particular $\operatorname{dim}_{s} A=m-1$;

3) $\left(A: A_{i}\right)$ is a radical ideal of $A_{i}$, for each $i, i=1, \cdots, m$.

Proof. Recall that by construction $A_{i+1}=\left(A_{i}: \mathscr{R}_{i}\right)$, for $i=0, \cdots$, $m-1$, and $\mathscr{R}_{i}$ is a strongly divisorial ideal of $A_{i}$. Thus to prove 1) it is enough to apply Lemma 3.1. To prove 2) observe that, by 1) and Proposition 1.10, $\operatorname{dim}_{s} A_{i+1}=\operatorname{dim}_{s} A_{i}-1$, for each $i, i=0, \cdots, m-1$. Recalling moreover that $A_{m}$ does not have strongly divisorial prime ideals, i.e. $\operatorname{dim}_{s} A_{m}=-1$, we get $\operatorname{dim}_{s} A_{i}=-1+(m-i)=m-i-1$. In particular $\operatorname{dim}_{s} A=\operatorname{dim}_{s} A_{0}=m-1$. To prove 3), we show that $A$ contains the radical of $\left(A: A_{i}\right)$ in $A_{i}$ for each $i, i=1, \cdots, m$. Let $x \in A_{i}$ 
and $x^{n} \in\left(A: A_{i}\right)$, for some $n \in N$. We want to prove that $x \in A$. It is enough to prove that $x \in A_{i-1}$ and $x^{n} \in\left(A: A_{i-1}\right)$. We have $\left(A: A_{i}\right) \subset$ $\left(A_{i-1}: A_{i}\right)=\mathscr{R}_{i-1}$, thus, since $\mathscr{R}_{i}$ is a radical ideal of $A_{i}, x \in \mathscr{R}_{i-1} \subset A_{i-1}$. Moreover, trivially, $x^{n} \in\left(A: A_{i}\right) \subset\left(A: A_{i-1}\right)$.

If $A$ is a Noetherian domain such that $\bar{A}=A^{*}$ is an $A$-module of finite type (i.e. $(A: \bar{A}) \neq(0)$ ), we shall prove that the particular case considered above (i.e. $\mathscr{R}_{i}$ radical ideal of $A_{i+1}$ in the sequence $(*)$ ) corresponds to seminormal case.

Recall that, given two rings $A \subset B, B$ integral over $A$, the seminormalization of $A$ in $B$ is the ring

$$
A_{B}^{+}=\left\{b \in B \mid b / 1 \in A_{\Re}+\operatorname{Rad}\left(S^{-1} B\right), \forall \Re \in \operatorname{Spec} A\right\}
$$

where $S=A \backslash \mathfrak{P}$ and $\operatorname{Rad}\left(S^{-1} B\right)$ is the Jacobson radical of $S^{-1} B$ (cf. [16]). It is well known that $A_{B}^{+}$is the largest subring $A^{\prime}$ of $B$ such that

i) for each $\mathfrak{B} \in \operatorname{Spec} A$, there is exactly one $\mathfrak{Q} \in \operatorname{Spec} A^{\prime}$ above $\mathfrak{B}$;

ii) the canoncal homomorphism $k(\mathfrak{P}) \rightarrow k(\mathfrak{\Omega})$ is an isomorphism. (cf. $[16,(1.1)])$.

Proposition 3.3. Let $A$ be a Mori domain and let $\mathfrak{P}_{1}, \cdots, \mathfrak{P}_{n} \in \mathscr{S}(A)$. If $\mathscr{R}=\mathfrak{P}_{1} \cap \cdots \cap \mathfrak{P}_{n}$ and $C=(A: \mathscr{R})$, then the following conditions are equivalent:

1) $\mathscr{R}$ is a radical ideal of $C$;

2) $S_{j}^{-1} \mathfrak{P}_{j}=\mathfrak{P}_{j} A_{\mathfrak{P}_{j}}$ is a radical ideal of $S_{j}^{-1} C$ (where $S_{j}=A \backslash \mathfrak{P}_{j}$ ), for each $j, j=1, \cdots, n$;

3) $A$ is the ring obtained from $C$ by glueing over $\mathfrak{\beta}_{1}, \cdots, \mathfrak{P}_{n}$.

Moreover, if $A$ is Noetherian, then the following are equivalent to each other and to the above conditions:

4) $A$ is seminormal in $C$;

5) $S_{j}^{-1} A=A_{\mathfrak{P}_{j}}$ is seminormal in $S_{j}^{-1} C$ (where $S_{j}=A \backslash \mathfrak{P}_{j}$ ), for each $j$, $j=1, \cdots, m$.

Proof. 1) $\Rightarrow 2)$ : since $\mathscr{R}$ is an ideal of $C, S_{j}^{-1} \mathscr{R}=S_{j}^{-1}\left(\mathfrak{P}_{1} \cap \cdots \cap \mathfrak{P}_{n}\right)$ $=S_{j}^{-1} \mathfrak{P}_{1} \cap \cdots \cap S_{j}^{-1} \mathfrak{P}_{n}=S_{j}^{-1} \mathfrak{R}_{j}$ is an ideal of $S_{j}^{-1} C$; since $\mathscr{R}$ is radical in $C, S_{j}^{-1} \mathfrak{P}_{j}$ is a radical ideal of $\left.\left.S_{j}^{-1} C .2\right) \Rightarrow 1\right): \mathscr{R}=\mathfrak{P}_{1} \cap \cdots \cap \mathfrak{P}_{n}=\mathfrak{P}_{1} A_{\mathfrak{P}_{1}} \cap$ $\cdots \cap \mathfrak{\beta}_{n} A_{\mathfrak{P}_{n}} \cap A$. By Proposition 1.13, $A=C \cap A_{\mathfrak{B}_{1}} \cap \cdots \cap A_{\mathfrak{P}_{n}}$, thus $\mathscr{R}=$ $\mathfrak{P}_{1} A_{\mathfrak{P}_{1}} \cap \cdots \cap \mathfrak{P}_{n} A_{\mathfrak{P}_{n}} \cap C$. Since $S_{j}^{-1} \mathfrak{P}_{j}$ is a radical ideal of $S_{j}^{-1} C, S_{j}^{-1} \mathfrak{P}_{j} \cap C$ is a radical ideal of $C$ for each $j, j=1, \cdots, n$, therefore $\mathscr{R}$ is a radical ideal of $C$. 2 ) $\Leftrightarrow 3$ ): by Proposition $2.7, A$ is the ring obtained from $C$ 
contracting $\mathfrak{P}_{1} C$ over $\mathfrak{P}_{1}, \mathfrak{P}_{2} C$ over $\mathfrak{P}_{2}, \cdots, \mathfrak{P}_{n} C$ over $\mathfrak{P}_{n}$. Thus $A$ is obtained by glueing over $\mathfrak{P}_{1}, \cdots, \mathfrak{P}_{n}$ if and only if $\mathfrak{\beta}_{1} C, \cdots, \mathfrak{P}_{n} C$ are radical ideals of $C$. This happens if and only if for each $j, j=1, \cdots, n, S_{j}^{-1} \mathfrak{P}_{j} C$ $=S_{j}^{-1} \mathfrak{P}_{j}$ is a radical ideal of $\left.S_{j}^{-1} C .2\right) \Rightarrow 5$ ): if $S_{j}^{-1} \mathfrak{P}_{j}$ is a radical ideal of $S_{j}^{-1} C$, necessarily $S_{j}^{-1} \mathfrak{P}_{j}=\operatorname{Rad}\left(S_{j}^{-1} C\right)$, the Jacobson radical of $S_{j}^{-1} C$, thus $S_{j}^{-1} A+\operatorname{Rad}\left(S_{j}^{-1} C\right)=S_{j}^{-1} A$ and $S_{j}^{-1} A$ is seminormal in $\left.S_{j}^{-1} C . \quad 5\right) \Rightarrow$ 4): observe that for each $j, j=1, \cdots, n$, the seminormalization of $A$ in $C$ is contained in the seminormalization of $S_{j}^{-1} A$ in $S_{j}^{-1} C$, as it follows by definition. Therefore we have $A_{C}^{+} \subset C \cap A_{\mathfrak{B}_{1}} \cap \cdots \cap A_{\mathfrak{P}_{n}}$. By Proposition 1.13, $C \cap A_{\mathfrak{R}_{1}} \cap \cdots \cap A_{\mathfrak{\Re}_{n}}=A$, thus $A$ is seminormal in $C$. 4) $\Rightarrow 1$ ): by [16, Lemma 1.3], because $\mathscr{R}$ is the conductor of $A$ in $C$.

Remark 3.4. Let $A$ be a Noetherian domain such that $\bar{A}$ is an $A$ module of finite type and let $B, C$ be two consecutive (Noetherian) domains of the associated sequence $(*)$. Proposition 3.3 gives, in particular, equivalent conditions in order that $B$ is seminormal in $C$.

Lemma 3.5. Let $A_{1} \subset A_{2} \subset B$ be domains and let $A_{2}=\left(A_{1}: \mathfrak{I}\right)$, where $\mathfrak{\Im}$ is a strongly divisorial ideal of $A_{1}$. If $\mathfrak{P} \in \operatorname{Spec} A_{2}, \mathfrak{P} \not \supset \mathfrak{S}, \mathfrak{p}=\mathfrak{P} \cap A_{1}$, $T_{1}=A_{1} \backslash \mathfrak{p}$ and $T_{2}=A_{2} \backslash \mathfrak{P}$, then $T_{1}^{-1} B=T_{2}^{-1} B$ and the ring obtained from $B$ by glueing over $\mathfrak{p} \in \operatorname{Spec} A_{1}$ coincides with the ring obtained from $B$ by glueing over $\mathfrak{B} \in \operatorname{Spec} A_{2}$.

Proof. Let's prove first that $T_{1}^{-1} B=T_{2}^{-1} B$. Let $x=b s^{-1} \in T_{2}^{-1} B$, with $b \in B, s \in T_{2}$. If $0 \neq i \in \mathfrak{S} \backslash \mathfrak{P}, b s^{-1}=(i b)(i s)^{-1} \in T^{-1} B$, because $i b \in B$, is $\in$ $\mathfrak{\Im} \subset A_{1}$ and $i \in A_{2} \backslash \mathfrak{R}, s \in A_{2} \backslash \mathfrak{B}$ so is $\notin \mathfrak{B} \cap A_{1}=\mathfrak{p}$. Thus $T_{1}^{-1} B \supset T_{2}^{-1} B$. The opposite inclusion is trivial. Let's prove now that $T_{1}^{-1} \mathfrak{p} B=T_{2}^{-1} \mathfrak{R} B$. Let $x=q b s^{-1}$, with $q \in \Re, b \in B, s \in T_{2}$. Pick as before an element $i \in$ $\mathfrak{S} \backslash \mathfrak{B}$. We have $x=b q i(s i)^{-1} \in T_{1}^{-1} \mathfrak{p} B$ because $q i \in \mathfrak{p}$ and $s i \in A_{1} \backslash \mathfrak{p}$. Thus $T_{1}^{-1} \mathfrak{p} B \supset T_{2}^{-1 \mathfrak{P} B}$. The opposite inclusion is trivial. Therefore $T_{1}^{-1} \sqrt{\mathfrak{p} B}=$ $\sqrt{T_{1}^{-1} \mathfrak{p} B}=\sqrt{T_{2}^{-1} \mathfrak{\beta} B}=T_{2}^{-1} \sqrt{\mathfrak{\beta} B}$. Recalling now that $\left(A_{1}\right)_{\mathfrak{p}}=\left(A_{2}\right)_{\mathfrak{\beta}}$ (cf. $[7,1.4, \mathrm{c})])$, we have that $k(\mathfrak{p})=k(\mathfrak{P})$ and, by definition of glueing, the conclusion.

Proposition 3.6. Let $A$ be a Mori domain such that $\left(A: A^{*}\right) \neq(0)$ and let $\left(^{*}\right)$ be the associated sequence. If, for each $i, i=0, \cdots, m-1$, $\mathscr{R}_{i}=\left(A_{i}: A_{i+1}\right)$ is a radical ideal of $A_{i+1}$ and if $\mathscr{S}\left(A_{i}\right)=\left\{\mathfrak{P}_{i 1}, \cdots, \mathfrak{P}_{i n(i)}\right\}$, then $A_{i}$ is the ring obtained from $A_{i+1}$ by glueing over $\mathfrak{p}_{i 1}=\mathfrak{B}_{i 1} \cap A, \cdots$, $\mathfrak{p}_{i n(i)}=\mathfrak{P}_{i n(i)} \cap A$. 
Proof. We already know according to Proposition $3.3,1) \Rightarrow 3$ ), that $A_{i}$ is the ring obtained from $A_{i+1}$ by glueing over $\mathfrak{P}_{i 1}, \cdots, \mathfrak{P}_{i n(i)}$. Observing that for each $j, j=1, \cdots, n(i), \Re_{i j} \not \supset\left(A: A_{i}\right)$ (cf. Lemma 3.1), and applying Lemma 3.5 we arrive at the conclusion.

Corollary 3.7. Let $A$ be a Mori domain such that $\left(A: A^{*}\right) \neq(0)$ and let $\left(^{*}\right)$ be the associated sequense. If, for each $i, i=0, \cdots, m-1, \mathscr{R}_{i}=$ $\left(A_{i}: A_{i+1}\right)$ is a radical ideal of $A_{i+1}$, then $A$ is obtained from $A^{*}$ by $a$ finite number of glueings over all the strongly divisorial prime ideals of $A$.

Proof. The Corollary follows immediately from Proposition 3.6. We have just to prove that the set $\{\mathfrak{p} \in \operatorname{Spec} A \mid \mathfrak{p}=\mathfrak{P} \cap A$ for some $i, i=$ $0, \cdots, m-1$, and some $\left.\Re \in \mathscr{S}\left(A_{i}\right)\right\}$ is the set of the strongly divisorial prime ideals of $A$. If $\Re \in \mathscr{S}\left(A_{i}\right)$ for some $i$, by Proposition 3.2, 3), $\left(A: A_{i}\right)$ is a radical ideal of $A_{i}$ and so, by Lemma $3.1, \mathfrak{P} \not \supset\left(A: A_{i}\right)$. Thus we can apply Proposition 1.10 and conclude that $\mathfrak{p}=\mathfrak{P} \cap A$ is a strongly divisorial ideal of $A$. On the other hand, let $\mathfrak{p}$ be a strongly divisorial prime ideal of $A$. If $\mathfrak{p} \notin \mathscr{S}(A)$, then $\mathfrak{p} \not \supset \mathscr{R}_{0}=\cap\{\mathfrak{Q} ; \mathfrak{Q} \in \mathscr{S}(A)\}=\left(A: A_{1}\right)$ and thus, again by Proposition 1.10 there exists in $A_{1}$ a strongly divisorial prime ideal $\mathfrak{p}_{1}$ over $\mathfrak{p}$. If $\mathfrak{p}_{1} \notin \mathscr{S}\left(A_{1}\right)$, then $\mathfrak{p}_{1} \not \supset \mathscr{R}_{1}=\left(A_{1}: A_{2}\right)$, thus there exists in $A_{2}$ a strongly divisorial prime ideal $\mathfrak{p}_{2}$ over $\mathfrak{p}_{1}$ (therefore over p) and so on. Since in $A_{m}$ there are not strongly divisorial prime ideals at all, there exist $i$ and $\mathfrak{B} \in \mathscr{S}\left(A_{i}\right)$ such that $\mathfrak{B} \cap A=\mathfrak{p}$.

TheOREm 3.8. Let $A$ be a Noetherian domain such that $\bar{A}$ is an $A$ module of finite type and let $(*)$ be the associated sequence. Then $A$ is seminormal if and only if $\mathscr{R}_{i}=\left(A_{i}: A_{i+1}\right)$ is a radical ideal of $A_{i+1}$, for each $i, i=0, \cdots, m-1$.

Proof. If $\mathscr{R}_{i}$ is a radical ideal of $A_{i+1}$ for each $i, i=0, \cdots, m-1$, then, by Proposition 3.3 and Remark 3.4. $A_{i}$ is seminormal in $A_{i+1}$. Thus, by [16, Lemma 1.2], we have that $A=A_{0}$ is seminormal in $\bar{A}=A_{m}$.

Conversely, let $A$ be seminormal (in $A_{m}=\bar{A}$ ). We want to prove that $A_{m-1}$ is seminormal in $A_{m}$. By Proposition 3.3 (and Remark 3.4), it is enough to show that, if $\mathfrak{B} \in \mathscr{S}\left(A_{m-1}\right)$, then $\mathfrak{B}\left(A_{m-1}\right)_{\mathfrak{B}}$ is a radical ideal of $S^{-1} A_{m}$ (where $S=A_{m-1} \backslash \mathfrak{R}$ ). Since, trivially, $A$ is seminormal in $A_{m-1}$, $\left(A: A_{m-1}\right)$ is a radical ideal of $A_{m-1}$ (cf. [16, Lemma 1.3]), so, by Lemma 3.1, $\mathfrak{P} \not \supset\left(A: A_{m-1}\right)$. Therefore we can apply Lemma 3.5 and, if $\mathfrak{p}=\mathfrak{B} \cap A$ and $T=A \backslash \mathfrak{p}$, we have $T^{-1} A_{m}=S^{-1} A_{m}$. Moreover $A_{\mathfrak{p}}=\left(A_{m-1}\right)_{\mathfrak{B}}$ and so 
$\mathfrak{p} A_{\mathfrak{p}}=\mathfrak{\beta}\left(A_{m-1}\right)_{\mathfrak{P}}$. Thus we have to show that $\mathfrak{p} A_{\mathfrak{p}}$ is a radical ideal of $T^{-1} A_{m}$. Observe now that, if $\mathfrak{S}=\left(A: A_{m}\right)$, since $\mathfrak{P} \supset\left(A_{m-1}: A_{m}\right) \supset \mathfrak{I}$, $\mathfrak{p} \supset \mathfrak{S}$. We claim that $\mathfrak{p}$ is a minimal over $\mathfrak{S}$. If not, we have $\mathfrak{\Im} \subset \mathfrak{q} \subsetneq \mathfrak{p}$, where $q$ is a strongly divisorial prime of $A$ (cf. Proposition 1.3). If this is the case, since $q \not \supset\left(A: A_{m-1}\right)$, by Proposition 1.10 , there is in $A_{m-1}$ a strongly divisorial prime ideal $\mathfrak{Q} \varsubsetneqq \mathfrak{P}$ and this is a contradiction, because $\operatorname{dim}_{s} A_{m-1}=0$ (cf. Proposition 3.2,2)). Thus $T^{-1} \mathfrak{J}=T^{-1} \mathfrak{p}$. Since $\mathfrak{I}$ is a radical ideal of $A_{m}$ (cf. again [16, Lemma 1.3]), $T^{-1} \tilde{\Im}=T^{-1} \mathfrak{p}=\mathfrak{p} A_{\mathfrak{p}}$ is a radical ideal of $T^{-1} A_{m}$.

Remark 3.9. As we recalled, if $A$ is seminormal, $(A: \bar{A})$ is a radical ideal of $\bar{A}$ (cf. [16, Lemma 1.3]). Observe that Theorem 3.8 provides, for a Noetherian domain $A$ such that $\bar{A}$ is an $A$-module of finite type, a kind of converse of this result. In order that $A$ is seminormal, it is not sufficient in general that the conductor $(A: \bar{A})$ is radical in $\bar{A}$, but it is sufficient (and necessary) that all the conductors $\mathscr{R}_{i}=\left(A_{i}: A_{i+1}\right), i=$ $0, \cdots, m-1$, of our sequence are radical in $A_{i+1}$. Trivially, if $m=1$ in the sequence (*), the two conditions $\left((A: \bar{A})\right.$ radical in $\bar{A}$ and $\mathscr{R}_{i}$ radical in $A_{i+1}$, for each $i$ ) are equivalent. A more general result in this spirit is the following:

Proposition 3.10. Let $A$ be a Mori domain such that $\left(A: A^{*}\right) \neq(0)$ and let $(*)$ be the associated sequence. If $\left(A: A^{*}\right)$ is a radical ideal of $A$ and if $\operatorname{dim}_{s} A=0$, then $m=1$, i.e. the sequence (*) is simply $A=A_{0} \subset$ $A_{1}=A^{*}$.

Proof. Since $\left(A: A^{*}\right)$ is radical, $\left(A: A^{*}\right)=\cap\left\{\mathfrak{P}_{\lambda} ; \lambda \in \Lambda\right\}$, where taking only the minimal primes over $\left(A: A^{*}\right)$, we can assume, by Proposition 1.3 , that all the $\mathfrak{P}_{2}$ are strongly divisorial primes of $A$. Since $\left(A: A^{*}\right)$ is the minimum strongly divisorial ideal of $A$ (cf. [3. Proposition 16]) and any intersection of strongly divisorial primes is a strongly divisorial ideal (cf. Proposition 1.2), it turns out that $\left(A: A^{*}\right)$ is the intersection of all the strongly divisorial primes of $A$. However, since by hypothesis there are not in $A$ non trivial chains of strongly divisorial primes, the set $\left\{\mathfrak{P}_{\lambda} ; \lambda \in \Lambda\right\}$ coincides with the set of all the strong maximal divisorial ideals of $A, \mathscr{S}(A)$ which, by Corollary 1.5 and since $\operatorname{dim}_{s} A=0$, is finite: $\left\{\mathfrak{P}_{1}, \cdots, \mathfrak{P}_{n}\right\}$. Thus $\left(A: A^{*}\right)=\mathfrak{P}_{1} \cap \cdots \cap \mathfrak{P}_{n}=\mathscr{R}_{0}$ and $A_{1}=\left(A: \mathscr{R}_{0}\right)=A^{*}$.

Remark 3.11. a) Notice that in Proposition 3.10 the hypothesis that ( $\left.A: A^{*}\right)$ is radical in $A$ is necessary, as Example 1.9, a) shows. 
b) If $A$ is a Mori domain such that $\left(A: A^{*}\right) \neq 0$, if $\left(^{*}\right)$ is the associated sequence, and if $\operatorname{dim}_{s} A=0$, we deduce easily from Proposition 3.10 that the following conditions are equivalent:

i) $\mathscr{R}_{i}=\left(A_{i}: A_{i+1}\right)$ is a radical ideal of $A_{i+1}$, for each $i, i=0, \cdots$, m-1;

ii) $\left(A: A^{*}\right)$ is a radical ideal of $A^{*}$.

In fact i) $\Rightarrow$ ii) is an easy consequence of Proposition 3.2 , 3) (recalling that $A_{m}=A^{*}$ ) and ii) $\Rightarrow \mathrm{i}$ ) is an easy consequence of Proposition 3.10, noticing that, if $\left(A: A^{*}\right)$ is radical in $A^{*}$, it is radical in $A$.

c) If $A$ is Noetherian, the equivalence of conditions i) and ii) above gives in particular the following known result: if $A$ is a Noztherian domain (with $A \neq(A: \bar{A}) \neq(0)$ ) which satisfies condition $\left(S_{2}\right)$ (depth $A_{\mathfrak{B}} \geq$ $\inf (2$, ht $\mathfrak{B})$, for all $\mathfrak{P} \in \operatorname{Spec} A)$, then $A$ is seminormal if and only if $(A: \bar{A})$ is a radical ideal of $\bar{A}$ (cf. [6 Proposition 7.12]). In fact $\left(S_{2}\right)$ holds in the Noetherian domain $A$ if and only if each $(0) \neq \Re \in \operatorname{Spec} A$, such that $\operatorname{depth} A_{\mathfrak{\beta}}=1$, is of height 1 , i.e., by [17, Proposition 1.10 , i) $\Leftrightarrow$ vi)], if and only if each divisorial prime of $A$ is of height 1 . However there is in $A$ at least one strongly divisorial prime, because $A(\neq \bar{A})$ is not a Krull domain (cf. [3, Corollary 14]), thus, if $\left(S_{2}\right)$ holds in $A, \operatorname{dim}_{s} A=0$. Moreover, if $A$ is Noetherian, condition i) above means that $A$ is seminormal (cf. Theorem 3.8).

Finally we point out that in the Mori, non-Noetherian case, the glueings over the strongly divisorial prime ideals of $A$ (of Corollary 3.7) do not request any algebraic or finiteness condition on the extension $k(\mathfrak{p})$ $\subset S^{-1} B / S^{-1} \mathfrak{J}$ (cf. Definition 2.1), as the simple following examples show:

ExAmples 3.12. a) Let $A=k+X k[X, Y]$ where $k$ is a field and $X, Y$ indeterminates over $k$, then $A$ is a Mori domain (cf. [4, Example (4.6), b)]). The associated sequence (*) is simply $A=A_{0} \subset A_{1}=A^{*}=k[X, Y]$ and $\left(A_{0}: A_{1}\right)=X k[X, Y]$ is a radical (in fact prime) ideal of $A^{*} . A$ is obtained from $A^{*}$ by glueing over $\mathfrak{p}=X k[X, Y]$. The transcendence degree 1 of the extension $k \subset k[Y]$ in the diagram

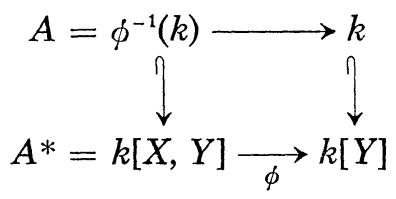

corresponds to the contraction of the affine line of generic point $X k[X, Y]$ $\in \operatorname{Spec} A^{*}$ to the point $\mathfrak{p}=X k[X, Y] \in \operatorname{Spec} A$. Outside of $\mathfrak{p}$, in the 
complement open set, $\operatorname{Spec} A$ and $\operatorname{Spec} A^{*}$ are scheme theoretically isomorphic.

b) Let $A=k[Z]+X Y k[X, Y, Z]$, where $k$ is a field and $X, Y, Z$ indeterminates over $k$. Then $A$ is a Mori domain, because $A=C \cap B_{1} \cap B_{2}$, where $C=k[X, Y, Z], \quad B_{1}=k(Z)+X k[X, Y, Z]_{(X)} \quad$ and $\quad B_{2}=k(Z)+$ $Y k[X, Y, Z]_{(Y)}$ are Mori domains (cf. [12, I, Theorem 2] and [2, Proposition 3.4]). The associated sequence (*) is simply $A=A_{0} \subset A_{1}=A^{*}=$ $k[X, Y, Z]$ and $\left(A_{0}: A_{1}\right)=X Y k[X, Y, Z]$ is a radical (non prime) ideal of $A^{*}$ (in fact $X Y k[X, Y, Z]=X k[X, Y, Z] \cap Y k[X, Y, Z]$ ). The domain $A$ is obtained from $A^{*}$ by glueing over $\mathfrak{p}=X Y k[X, Y, Z]$.

The two affine planes of generic points $\mathfrak{P}_{1}=X k[X, Y, Z]$ and $\mathfrak{P}_{2}=$ $Y k[X, Y, Z]$ of $\operatorname{Spec} A^{*}$ are identified in $\operatorname{Spec} A$ in the affine line of generic point $\mathfrak{p}$. Outside of $\mathfrak{p}$, in the complement open set, $\operatorname{Spec} A$ and Spec $A^{*}$ are scheme theoretically isomorphic.

c) Let $A=k+X k[X]+X Y k[X, Y, Z]$, where $k$ is a field and $X, Y, Z$ indeterminates over $k$. Then $A$ is a Mori domain, because it is not difficult to show that $A=C \cap B_{1} \cap B_{2}$, where $C=k[X, Y, Z], B_{1}=k(Z)+$ $X k[X, Y, Z]_{(X)}$ and $B_{2}=k(X)+Y k[X, Y, Z]_{(Y)}$ are Mori domains (cf. [12, 1, Theorem 2] and [2, Proposition 3.4]). Since $\mathfrak{p}_{1}=X k[X, Y, Z]_{(X)} \cap A=$ $X k[X]+X Y k[X, Y, Z] \supset \mathfrak{p}_{2}=Y k[X, Y, Z]_{(Y)} \cap A=X Y k[X, Y, Z]$, by $[4$, Theorem (4.3)], $\left\{\mathfrak{p}_{1}\right\}=\mathscr{S}(A)$, and the associated sequence $\left(^{*}\right)$ is $A=A_{0} \subset$ $A_{1}=k[X]+Y k[X, Y, Z] \subset A_{2}=A^{*}=k[X, Y, Z] . \quad\left(A_{0}: A_{1}\right)=X k[X]+$ $X Y k[X, Y, Z]$ is a prime ideal of $A_{1}$ and $\left(A_{1}: A_{2}\right)=Y k[X, Y, Z]$ is a prime ideal of $A^{*}$. Thus $A$ is obtained from $A^{*}$ by glueing over the strongly divisorial prime ideals of $A, \mathfrak{p}_{1}$ and $\mathfrak{p}_{2}$. The affine plane of generic point $\mathfrak{P}_{1}=X k[X, Y, Z]$ of $\operatorname{Spec} A^{*}$ is contracted in Spec $A$ into the point $\mathfrak{p}_{1}$; the affine plane of generic point $\mathfrak{P}_{2}=Y k[X, Y, Z]$ of Spec $A^{*}$ is contracted in Spec $A$ into the affine line of generic point $\mathfrak{p}_{2}$. Since $\left(A: A^{*}\right)=\mathfrak{p}_{2}$, outside of $\mathfrak{p}_{2}$, in the complement open set, $\operatorname{Spec} A$ and $\operatorname{Spec} A^{*}$ are scheme theoretically isomorphic.

\section{REFERENCES}

[1] M. F. Atiyah-I. G. Macdonald, Introduction to Commutative Algebra, AddisonWesley, Reading, Mass., 1969.

[2] V. Barucci, On a class of Mori domains, Comm. Algebra, 11 (17) (1983), 1989-2001.

[ 3 ] - Strongly divisorial ideals and complete integral closure of an integral domain, J. Algebra, 99 (1) (1986), 132-142.

[4] V. Barucci - S. Gabelli, How far is a Mori domain from being a Krull domain?, J. Pure Appl. Alg., 45 (1987), 101-112. 
[5] - On the class group of a Mori domain, J. Algebra, 108 (1) (1987), 161-173.

[6] M. Bass - M. P. Murthy, Grothendieck groups and Picard groups of abelian group rings, Ann. of Math., 86 (1967), 16-73.

[7] M. Fontana, Topologically defined classes of commutative rings, Annali Mat. Pura Appl., 123 (1980), 331-355.

[ 8 ] R. Fossum, The divisor class group of a Krull domain, Springer-Verlag, 1973.

[ 9 ] F. Ischebeck, Zwei Bemerkungen über seminormale Ringe, Math. Z., 152 (1977), 101-106.

[10] J. Lipman, Stable ideals and Arf rings, Amer. J. Math., 93 (1971), 649-685.

[11] J. Querré, Sur une propriété des anneaux de Krull, Bull. Sc. Math., 95 (1971), 341-354.

[12] N. Raillard (Dessagnes), Sur les anneaux de Mori, C. R. Acad. Sc. Paris, 280 (1975) , 1571-1573.

[13] _- Sur les anneaux de Mori, thèse, Univ. Pierre et Marie Curie, Paris VI, 1976.

[14] G. Tamone, Sugli incollamenti di ideali primari e la genesi di certe singolarità, B.U.M.I. (Supplemento) Algebra e Geometra, Suppl., 2 (1980), 243-258.

[15] — A connection between blowing-up and glueings in one-dimensional rings, Nagoya Math. J., 94 (1984), 75-87.

[16] C. Traverso, Seminormality and Picard group, Ann. Sc. Norm. Sup. Pisa, 24 (4) (1970), 585-595.

[17] K. Yoshida, On birational-integral extension of rings and prime ideals of depth one, Japan J. Math., 8 (1982), 49-70.

Dipartimento di Matematica

Istituto "G. Castelnuovo"

Università di Roma "La Sapienza"

Piazzale A. Moro 5, 00185 Roma

Italia 